\title{
Controllable Data Synthesis Method for Grammatical Error Correction
}

\author{
Liner Yang ${ }^{\S}$, Chencheng Wang ${ }^{\dagger}$, Yun Chen ${ }^{\ddagger}$, Yongping Du ${ }^{\dagger}$, Erhong Yang $^{\S}$ \\ ${ }^{\S}$ Beijing Language and Culture University, Beijing, China \\ ${ }^{\dagger}$ Beijing University of Technology, Beijing, China \\ ${ }^{\ddagger}$ Shanghai University of Finance and Economics, Shanghai, China \\ \{lineryang, hsamswang, yun. chencreek\} @gmail.com \\ ypduabjut.edu.cn, yerhong@blcu.edu.cn
}

\begin{abstract}
Due to the lack of parallel data in current Grammatical Error Correction (GEC) task, models based on Sequence to Sequence framework cannot be adequately trained to obtain higher performance. We propose two data synthesis methods which can control the error rate and the ratio of error types on synthetic data. The first approach is to corrupt each word in the monolingual corpus with a fixed probability, including replacement, insertion and deletion. Another approach is to train error generation models and further filtering the decoding results of the models. The experiments on different synthetic data show that the error rate is $40 \%$ and the ratio of error types is the same can improve the model performance better. Finally, we synthesize about 100 million data and achieve comparable performance as the state of the art, which uses twice as much data as we use.
\end{abstract}

\section{Introduction}

The task of Grammatical Error Correction is primarily aimed at detecting and correcting errors in essays. e.g. I follows his advices $\rightarrow$ I followed his advice. With the increasing number of English as a Second Language learners (ESL) and the success of four shared tasks (Dale and Kilgarriff, 2011; Dale et al., 2012; Ng et al., 2013, 2014), more and more researchers focus on the study of GEC.

Since Brockett(Brockett et al., 2006) introduced the idea of translation to solve the problem of GEC by translating error text into right one, a lot of sequence to sequence models have been applied to GEC (Chollampatt and Ng, 2018a,b; Grundkiewicz and Junczys-Dowmunt, 2018; Ge et al., 2018). All of these studies are based on two public corpora, the largest parallel corpus Lang-8 (Mizumoto et al., 2011) and NUS Corpus of Learner English (NUCLE) (Dahlmeier et al., 2013).
Although these corpora contain millions of sentence pairs, the model still cannot be trained to achieve higher performance in GEC tasks. Thus, the state-of-the-art systems (Junczys-Dowmunt et al., 2018; Zhao et al., 2019; Lichtarge et al., 2019) regard GEC as a low-resource Neural Machine Translation (NMT) task. Neither of their methods takes full account of the impact of different error rates and error type ratios on the results. Therefore, we propose two data synthesis methods that can control the error information of synthetic data. The effects of different error rates, error types and the size of the synthesized corpus on the performance of the model are also discussed.

The first approach is rule-based, corrupting each token of monolingual corpus with a fixed probability according to the required error rate. Corruption operations include deleting tokens, inserting and replacing random tokens in the vocabulary. The ratio of error types on the synthetic data is controlled by the probability of operations.

The second approach is based on Back translation mechanism. We separately train statistical machine translation (SMT) and NMT models on the corpora of correct $\rightarrow$ error sentence pairs and introduce noise to the monolingual corpus in the decoding stage. In order to control the error distribution of synthetic data, we propose a filtering strategy to remove instances with lower error rate and types of errors that we don't need.

By controlling variables, we explore the effects of different error rates and ratio of error types on the model performance. Finally, we pretrain our model on 100 million synthesized data and achieve a comparable result to the state of the art (Lichtarge et al., 2019), which use about 170 million Wikipedia revision histories with manual modifications. 


\section{Method}

\subsection{GEC system}

A lot of recent work has proved the feasibility of using Sequence-to-Sequence Model to solve GEC problems, which showed immense success in neural machine translation. Unlike NMT task, there are not enough available learner corpora in GEC. This motivates the use of data augmentation with monolingual data.

Figure 1 shows the process of our GEC system. We introduce two important concepts and propose two approaches for data augmentation, including ruled-based method and Back-translation. Furthermore, we put forward a filtering strategy for the corpora to balance error rate and ratio of error types. After pre-training on the synthetic parallel corpora, we fine-tune our GEC model with learner corpora.

\subsection{Error Rate}

Although the task of grammatical error correction is currently regarded as the translation task, the main difference is that both source and target of the GEC use the same language. The original text and its corresponding correction text may be very similar or different. Two examples are shown in the following:

(1) Error : Students often travel hundreds of mile to get here.

Correct : Students often travel hundreds of miles to get here.

(2) Error : Students often travel to here .

Correct : Students often travel hundreds of miles to get here .

The first pair of sentences has few modifications. When the model is over-trained on such data, it is more likely to simply copy the inputs without correcting any words. For the second example, the error sentence contains too little information to be corrected. Models can hardly learn useful information from the training data like this. It is feasible to train decoders with such examples (Sennrich et al., 2016a), but this is beyond the scope of this work.

Therefore, we hope to quantitatively analyze the amount of information contained in the corpus. We define the Error Rate $\left(E_{\text {rate }}\right)$ in grammar er- ror correction task as:

$$
E_{\text {rate }}(S)=\frac{\sum_{i=0}^{n} \text { Levenshtein }\left(S_{i}^{\text {err }}, S_{i}^{c o r}\right)}{\sum_{i=0}^{n} \operatorname{Length}\left(S_{i}^{c o r}\right)}
$$

where $n$ is the number of sentence pairs in the corpus $S, S^{\text {err }}$ refers to the source sentence and $S^{c o r}$ is the corresponding correction. Levenshtein $\left(S_{i}^{\text {err }}, S_{i}^{\text {cor }}\right)$ is the shortest edit distance (minimum number of replacements, insertions and deletions) between $S_{i}^{\text {err }}$ and $S_{i}^{\text {cor }}$ in token level. Length $\left(S_{i}^{\text {cor }}\right)$ denotes the number of tokens in $S_{i}^{c o r}$.

\subsection{Error Category}

Another important concept in GEC task is the error types. We use the error categories proposed by Bryant (Bryant et al., 2017) and further divide them into two kinds of granularity.

(1) Coarse-grained error category :

- Missing type (M) $\begin{array}{llllll}\text { err }: \mathrm{w}_{0} & \ldots & \mathrm{w}_{i-1} & \mathrm{w}_{i+1} & \ldots & \mathrm{w}_{n}\end{array}$ $\begin{array}{llllllll}\text { cor: }: \mathrm{w}_{0} & \ldots & \mathrm{w}_{i-1} & \mathbf{w}_{\boldsymbol{i}} & \mathrm{w}_{i+1} & \ldots & \mathrm{w}_{n}\end{array}$ Here, $\mathbf{w}_{i}$ is a missing error type.

- Unnecessary type (U) $\begin{array}{llllllllll}\text { err: }: \mathrm{w}_{0} & \ldots & \mathrm{w}_{i-1} & \mathbf{w}_{\boldsymbol{i}} & \mathrm{w}_{i+1} & \ldots & \mathrm{w}_{n}\end{array}$ cor: $\begin{array}{llllll}\mathrm{w}_{0} & \ldots & \mathrm{w}_{i-1} & \mathrm{w}_{i+1} & \ldots & \mathrm{w}_{n}\end{array}$ Here, $\mathbf{w}_{i}$ is an unnecessary error type.

- Replacement type $(\mathbf{R})$ $\begin{array}{lllllll}\text { err: }: \mathrm{w}_{0} & \ldots & \mathrm{w}_{i-1} & \mathbf{w}_{\boldsymbol{i}}^{\prime} & \mathrm{w}_{i+1} & \ldots & \mathrm{w}_{n}\end{array}$ $\begin{array}{llllllll}\text { cor: }: \mathrm{w}_{0} & \ldots & \mathrm{w}_{i-1} & \mathbf{w}_{\boldsymbol{i}} & \mathrm{w}_{i+1} & \ldots & \mathrm{w}_{n}\end{array}$ Here, $\mathbf{w}_{i}$ is a replacement error type.

(2) Fine-grained error category :

We distinguish 25 different error types described in (Bryant et al., 2017). Such as, ADJ : adjective error, ADV : Adverb error, DET : determiner error and so on.

\subsection{Controllable Rule-based Corpora Corruption}

Increasing the size of the training set is a common and effective method to improve performance of neural network. By introducing artificial errors to monolingual corpus, we can get more rich training data for different topic and language phenomenon with the error types we need.

Previous study synthesized data containing determiner and noun number errors and found that the recall on these error types increased significantly, but the recall on other errors declined (Xie 


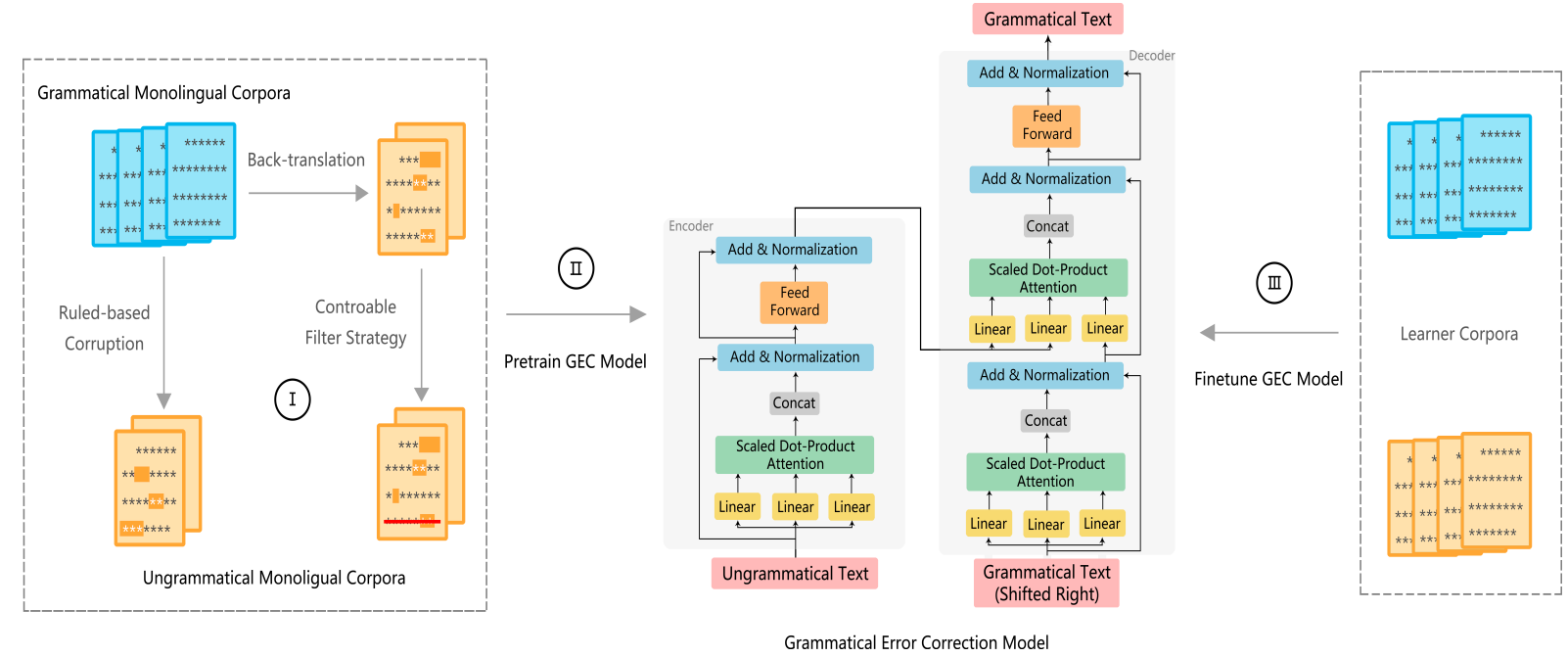

Figure 1: The Grammatical Error Correction system. (I) Introducing errors into grammatical monolingual corpora in two ways. (II) Using synthetic parallel corpora to pretrain the model. (III) Finetuning the GEC model with learner corpora.

\begin{tabular}{|c|c|}
\hline Error Rate & Output \\
\hline $0 \%$ (original) & $\begin{array}{l}\text { Results from dozens of clinical studies will be released at the gathering opening Friday and } \\
\text { running through June } 2 \text {. }\end{array}$ \\
\hline $10 \%$ & $\begin{array}{l}\text { Results from dozens of clinical studies will [miss] released at the gathering opening Friday } \\
\text { and Overmedicated running through June } 2 \text {. }\end{array}$ \\
\hline $30 \%$ & $\begin{array}{l}\text { Results from dozens lifestyles of [miss] studies will be released at [miss] gathering opening } \\
\text { Friday and running through [miss] . }\end{array}$ \\
\hline $50 \%$ & $\begin{array}{l}\text { Results from [miss] will be [miss] at gathering Friday skeptical and running Alyas through } \\
\text { alternative June in } 2 \text { ! }\end{array}$ \\
\hline
\end{tabular}

Table 1: Examples of the rule-based corruption with different Error Rates. The Bold Font represents the difference from the original text. [miss] means that the token is deleted.

et al., 2016). Therefore, only increasing partial error types may lead to the deviation of model.

In order to control the error rate and the ratio of error types on synthetic data, we apply the same processing method to each sentence in One Billion Word Benchmark monolingual corpus (Chelba et al., 2013). This corpus consist of nearly one billion words of English taken from news articles on the web. We corrupt each token $t$ with a fixed probability $E_{\text {rate }}$. The operations of corruption include, deleting the current token $t$ with a probability of $p_{m}$ (Missing error type), inserting a random token $t^{\prime}$ in the vocabulary $V$ to the left of $t$ with a probability of $p_{u}$ (Unnecessary error type) and replacing $t$ with a random token $t^{\prime}$ in $V$ with a probability of $p_{r}$ (Replacement error type). Each operation corresponds to an error in this sentence pair. When the replaced token $t \in \mathbb{P}=\left\{\prime^{\prime}, \ldots\right.$ ! ? $\}$, it can be only replaced by the elements in $\mathbb{P}$. Algorithm 1 formalizes this ap- proach. The vocabulary $V$ is a collection of all different tokens in the monolingual and labeled corpora.

Examples of the corruption are shown in Table 1. It shows that the result of corruption is very similar to the original text with the low $E_{\text {rate }}(10 \%)$. With the increasing of $E_{\text {rate }}$, the outputs of corruption become more disorderly.

\subsection{Corpus Generation Based on Back-translation}

For fine-grained error category, it is very difficult to define corrupt rules for all of the 25 error types. This is not only time-consuming, but also difficult to cover all the error phenomena wrote by learners. Therefore, we adopt the Back-translation mechanism for synthesizing error text set. The main idea of this mechanism is to train an error generation model on the corpus of correct $\rightarrow$ error sentences, which is the opposite of the error correction model. In the decoding stage, model gen- 


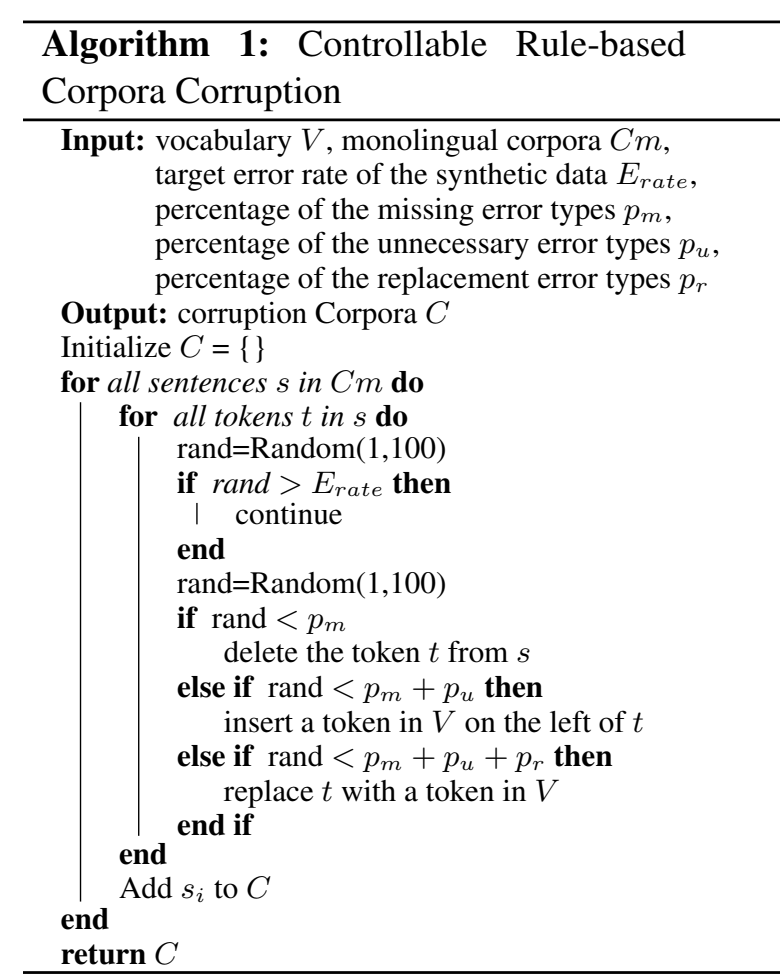

erates error sentences by introducing noise into monolingual data.

We implement the error generation models in two ways, include phrase based Moses system ${ }^{1}$ (Koehn et al., 2007) and attention based neural network Transformer (Vaswani et al., 2017). Details of the implementation are shown as follows.

\section{- Moses-Based error generation model}

We don't use the preprocessing script provided by Moses Open Source Library on the labeled data. We use GIZA $++^{2}$ for word-aligning our parallel data. The KenLM ${ }^{3}$ is used for training a trigram language model on the source sentences of the labeled data. The development set is used to fine-tune the model.

- Transformer-Based error generation model We use subword (Sennrich et al., 2016b) algorithm to train a Byte Pair Encoding(BPE) vocabulary on labeled data. The labeled and monolingual data is split into sub-words by BPE vocabulary. The union of sub-words is the vocabulary used in error generation model. We evaluate the development set at the end of each epoch and save the model checkpoint with the best cross entropy cost. The settings of hyper parameters are the same as

\footnotetext{
${ }^{1} \mathrm{http} / / / \mathrm{www}$. statmt.org/moses/?n=Moses.Baseline

${ }^{2}$ https://github.com/moses-smt/giza-pp

${ }^{3}$ https://kheafield.com/code/kenlm/estimation/
}

the error correction model (described in section 4.2). We follow the recommendation by Edunov (Edunov et al., 2018) to restrict sampling over the ten most likely words during inference stage.

The proposed error generation models based on Moses and Transformer are trained on Lang8 and NUCLE. The development set is CoNLL2013 test set. Examples of the Back-translation are shown in Table 2. It can be seen that the decoding results are conservative. Compared with the original sentence, the outputs contain only a few errors.

\subsection{Filtering Strategy for synthetic data}

In the previous section, we find that data synthesized by the Back-translation method contains lower error rate. Training on a corpus with fewer errors will make the models become more conservative when correcting errors. To solve this problem, we propose a filter strategy for the synthetic data.

The goal of the strategy is to balance error rate and ratio of error types. We greedily remove sentence pairs with lower error rate and those containing error types we do not need. The steps are shown in the following.

1) Given the synthetic data $X$ and corresponding corrected data $Y$, target error rate $E_{\text {rate }}$, target ratio of $n$ error types EtRatio = $\left\{e_{1}, e_{2}, \ldots, e_{n}\right\}$ and the threshold $\theta$ which controls the accuracy of filtering.

2) Calculate the error rate of each sentence pair and arrange them in ascending order.

3) Remove sentence pairs greedily until error rate of $E_{\text {rate }} *(1-\theta)$ is achieved.

4) Calculate the $n$ error types for each sentence pairs ${ }^{4}$ and count them as $E t=$ $\left[\right.$ num $_{1}$, num $_{2}, \ldots$, num $\left._{n}\right]$.

5) Count the sum of $n$ error types in the synthetic data and record them as EtSum = $\left\{\right.$ sum $_{1}$, sum $_{2}, \ldots$, sum $\left._{n}\right\}$.

6) Find the subscript $i$ for the lowest target error type ratio EtRatio[i], mark the number EtSum $[i]$ as benchmark B. The benchmark is the number of output for each proportion.

7) Using algorithm 2 to filter out sentence pairs that contain error types beyond the target ratio.

\footnotetext{
${ }^{4}$ https://github.com/chrisjbryant/errant/blob/master/parallel _to_m2.py
} 


\begin{tabular}{|c|c|}
\hline Method & Output \\
\hline Original & $\begin{array}{l}\text { Forecasts called for plunging temperatures and afternoon rain storms in the early Southern } \\
\text { Hemisphere autumn. }\end{array}$ \\
\hline Moses & $\begin{array}{l}\text { Forecasts called for plunging temperature and afternoon rain storm in the early Southern } \\
\text { Hemisphere autumn [miss] }\end{array}$ \\
\hline Transformer & $\begin{array}{l}\text { Forecasts called [miss] plunging temperatures and afternoon rain storm in the early South } \\
\text { Hemisphere autumn. }\end{array}$ \\
\hline
\end{tabular}

Table 2: Examples of the Back-translation with different models. The Bold Font represents the difference from the original text. [miss] means that the token is deleted.

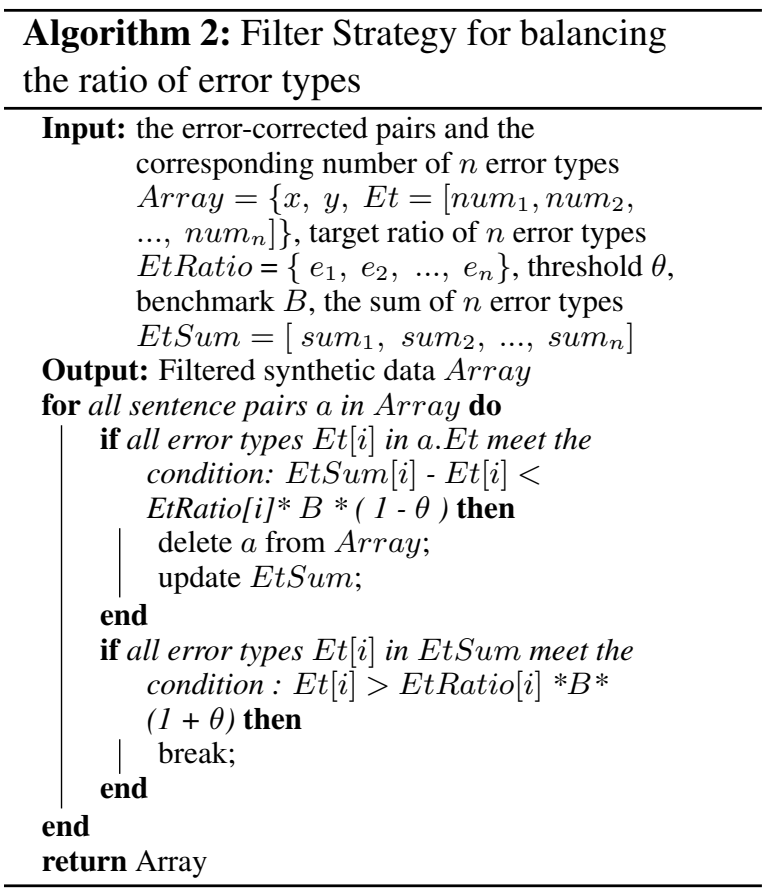

\section{Experiment Setup}

\subsection{Datasets}

We use NUCLE (Dahlmeier et al., 2013) and Lang8 (Mizumoto et al., 2011) as our labeled training data. We choose the test set from the CoNLL 2013 shared task (Ng et al., 2013) as our development set and report MaxMatch(M2) score (Dahlmeier and Ng, 2012) on the CoNLL-2014 shared task (Ng et al., 2014) test set. The monolingual corpus we use is the One Billion Word Benchmark (Chelba et al., 2013).

We remove the uncorrected sentence pairs from the labeled data like previous work (Zhao et al., 2019). By analyzing the data, we find that there are many instances containing URLs in NUCLE, illegal characters and emoji in Lang8. We remove the sentence pairs containing these cases from all training sets. The statistics of the corpora after preprocessing are given in Table 3.

\begin{tabular}{ccc}
\hline Corpus & \#sentences & \#tokens \\
\hline NUCLE & 21,177 & $0.5 \mathrm{M}$ \\
Lang-8 & $1,230,095$ & $18.5 \mathrm{M}$ \\
One-Billion* & $19,966,137$ & $497 \mathrm{M}$ \\
CoNLL2013 & 1,382 & - \\
CoNLL2014 & 1,312 & - \\
\hline
\end{tabular}

Table 3: Statistics for the sentence pairs of all data after preprocessing. Here, * refers that this corpus is unlabeled.

\subsection{Hyper Parameters}

In this work, we use the Transformer model (Vaswani et al., 2017) implemented by FAIR ${ }^{5}$ tool kits as the error correction model.

The detailed parameters of the model are as follows: Both of the source embedding and the target embeddings have 512 dimensions and use the same BPE vocabulary. We share the weights of decoder's input and output embeddings. Both of the encoder and decoder have 6 multi-head layers and 8 attention heads. The size of inner layer at each multi-head layer is 2048. We use Adam optimizer to train transformer model with the inverse squared root schedule which will decay the learning rate based on the inverse square root of the warm-up steps. The learning rate initialize with $5 \times 10^{-4}$ and warm-up during the first 4,000 steps. In order to train transformer adequately, we use a batch size of 32,000 tokens and fine-tune the model on labeled data for 30,000 steps. Dropout is applied at a ratio of 0.3. The Loss function we use is the Edit-weighted MLE objective (JunczysDowmunt et al., 2018) and the factor $\Lambda$ is set to 1.2.

We use a random seed value of 3 for training process and save the model checkpoints for each epoch. The ensemble model consists of eight checkpoints that get the best loss value on devel-

\footnotetext{
${ }^{5}$ https://github.com/pytorch/fairseq
} 
opment set during fine-tuning. Calculate the geometric average (Lichtarge et al., 2019) of all single model outputs as the final output of the ensemble model. During inference stage, we set beam size to 12 and keep the best decoding results as the model output without using any re-ranking.

\section{Experiment and Analysis}

As mentioned in Junczys-Dowmunt, Sequence-toSequence training of neural networks is a recognition optimization process, so it is prone to instability (Junczys-Dowmunt et al., 2018). We use different random seeds to initialize the model. Table 4 shows that different initialization weights have a great influence on the performance of the model. Therefore, we will report the average of the model scores initialized by random seed $=1,2$ and 3 (not ensemble) in all exploratory experiments.

\begin{tabular}{cccc}
\hline Seed & Precision & Recall & $\mathbf{F}_{\mathbf{0 . 5}}$ \\
\hline 1 & 57.44 & 32.25 & 49.68 \\
2 & 55.56 & 30.80 & 47.86 \\
3 & 56.67 & 32.19 & 49.19 \\
\hline
\end{tabular}

Table 4: Scores of models initialized with different random seeds.

We will analyze the impact of different error rates and ratio of error types on synthetic data and also verify the effectiveness of the filtering strategy. We use the ERRANT ${ }^{6}$ to evaluate the model performance by different error types.

\subsection{Performance impact by different Error Rates}

Firstly, we investigate the impact of different error rates by the synthetic data on model performance. We corrupt 1.25 million (1:1 to the labeled data) monolingual corpus by rule-based method described in Section 3.3. The ratio of the insertion, deletion and replacement operations is 1:1:1.

We generate 10 sets of synthetic data with different error rates range from $10 \%$ to $100 \%$. Each set of the synthetic data is trained for five epochs. Then, the labeled data is performed $30 \mathrm{~K}$ updates during fine-tuning. The other parameters of the model used in the pre-training stage are the same as those in the fine-tuning stage (described in Section 4.2). We record the scores of three models initialized with random seed $=1,2,3$ and report the average of them (The blue line in Figure 2(a)).

\footnotetext{
${ }^{6}$ https://github.com/chrisjbryant/errant
}

We also train a baseline model that only use the labeled data without pre-training (The red line in Figure 2(a)).
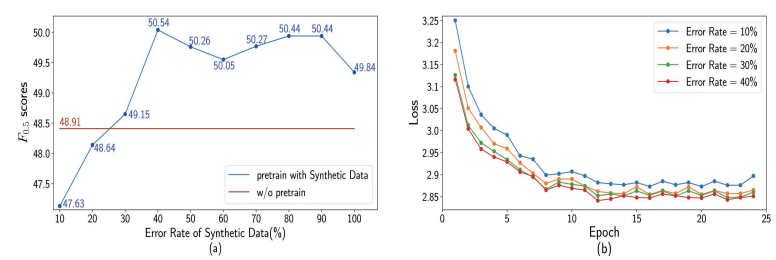

Figure 2: (a) $\mathrm{F}_{0.5}$ scores of models pre-training on the synthetic data with different error rates corrupted by rule-based method. The red line is the baseline model training on the labeled data. The blue line is the models pre-training on the different synthetic data and finetuning with the labeled data. (b) The loss value variation of four fine-tuning models which are pre-trained on synthetic data with different error rate.

Figure 2(a) shows that pre-training on the synthetic data with error rate less than $30 \%$ is unhelpful to improve the performance of the error corrected model. When training on the synthetic data with few mistakes $(<=10 \%)$, the performance is declined greatly compared with the baseline. This also confirms what Xie said, "the addition of examples where source and target are identical data may also cause the model to become too conservative with edits and thus reduce the recall of the system" (Xie et al., 2018). Therefore, we should avoid using synthetic data with low error rate, even labeled data.

The model performance gets best when error rate is $40 \%$. With the increasing of error rate $(>=40 \%)$ on synthetic data, the scores of the model do not change significantly. However, it is worth noting that, pre-training with the high error rate $(>=80 \%)$ is still helpful to improve the model performance. Theoretically, the source of these data should be extremely disorderly. But experiments show that the error corrected model can still learn useful information from the noisy source.

Figure 2(b) shows the variation of loss value for models pretraining on synthetic data with different error rate. The starting point of the four lines represents the superiority of the pre-training results for synthetic data with different error rate. Pretraining GEC model using synthetic corpus with error rate of $40 \%$ (red line in (b)) can make the model initialized into a more reasonable parameter space. The effect of pre-training still has a great impact on the process of fine-tuning, since we can 
see that the red line (Error Rate $=40 \%$ ) has been kept at a very low value in the Figure 2(b).

\subsection{Performance impact by different ratio of Error Types}

To make a comparison with the previous experiments, we still generate 1.25 million data with the rule-based methods and set $E_{\text {rate }}$ to $30 \%$. We vary the proportion of the three operations, $p_{m}=20 \%$, $p_{u}=20 \%, p_{r}=60 \%$ as the first group of experiments recorded as 1:1:3. The other two groups are, $p_{m}=60 \%, p_{u}=20 \%, p_{r}=20 \%$ and $p_{m}=20 \%, p_{u}=60 \%, p_{r}=20 \%$ denoted as $3: 1: 1$ and 1:3:1, respectively. The procedure of pre-training synthetic data is the same as before.

Figure 3 summarizes the results about these experiments. We separately evaluate the $\mathrm{F}_{0.5}$ score of coarse-grained error category, $\operatorname{Missing}(\mathrm{M})$, Unnecessary $(\mathrm{U})$ and $\operatorname{Replacement}(\mathrm{R})$. The performance of 3:1:1 group, which has more missing type errors, shows a significant increase in $M$ type compared with 1:1:1. The group of 1:3:1 performs well in $U$ type and it is as expected.

Unexpectedly, the experiment of 1:1:3 gets the worst result and does not improve in $\mathrm{R}$ type. The model may not understand the replacement operations very well. In terms of the results, adding more replacement errors of synthetic data just makes the imodel learn better on missing and unnecessary type errors. Because a replacement operation consists of an insert and a delete operation.
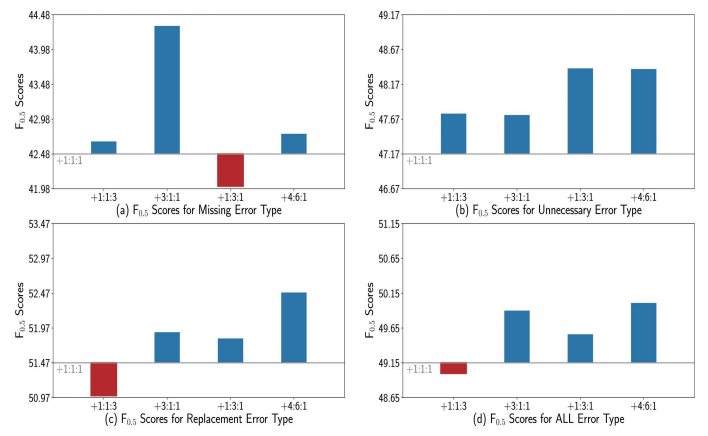

Figure 3: Scores of models pre-training on the synthetic data corrupted by rule-based method with different ratio of error types. (a), (b), (c) and (d) represent the $\mathrm{F}_{0.5}$ scores for Missing, Unnecessary, Replacement and ALL error type, respectively.

The ratio of $M: U: R$ in the original training set is $29: 12: 59$ and the error rate is approximate $34 \%$. We generate a set of data with $E_{\text {rate }}=30 \% p_{m}=$ $36 \% p_{u}=55 \% p_{r}=9 \%(4: 6: 1)$, which makes the M:U:R on the total data to $1: 1: 1$. The model achieves the best score 50.01 .

\subsection{Performance impact by different size of Synthetic Data}

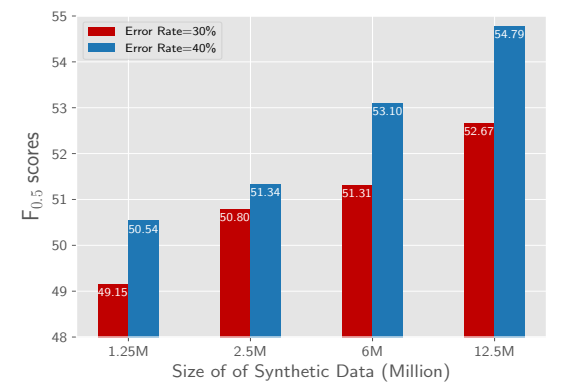

Figure 4: $\mathrm{F}_{0.5}$ scores of models pre-training on the synthetic data with different number of synthetic data. Each error rate contains four sets of experiments, including 1:1 (1.25M), 2:1 (2.5M), 4:1 (6M) and 10:1 (12.5M).

In order to investigate whether performance improvement is increasing with higher ratios of synthetic data, we perform four experiments for different error rate of synthetic data.

Figure 4 shows that when the size of the synthetic data is the same, the corpus with $40 \%$ error rate contributes more to the model than the corpus with $30 \%$ error rate. As the scale of synthetic data increasing, the performance of the model becomes better and better. This conflicts with the conclusion of Fadaee et al. (Fadaee and Monz, 2018) in machine translation task. They find that translation quality does not improve linearly with the size of the synthetic data. The model trained on (1:4) real to synthetic ratio of training data achieves the best results.

In our opinion, the main reason for the different conclusions is the particularity of grammatical error correction task. Since the source and target language is the same in GEC, we can regard it as a denoising procedure. The pseudo-data generated by adding noise to rich contexts can be used to improve the denoising ability of the model.

\subsection{Effectiveness of the Filtering Strategy}

The goal of the filtering strategy is to control error rate and ratio of error types in corpus. In previous experiments, we have found that adjusting the error rate or error types of synthetic corpus has a great influence on model performance. Therefore, we further verify that the filtering strategy for fine- 
grained error types is also effective for data generated by Back-translation.

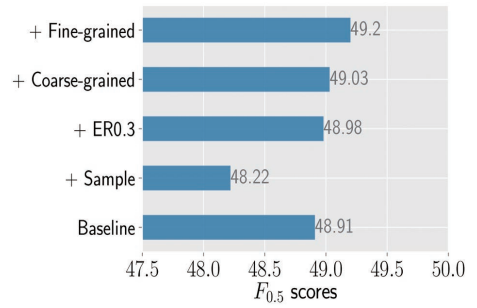

Figure 5: Scores of models pre-training on the synthetic data generated by Back-translation. Baseline refers the model training on labeled data without pretraining.

The two methods described in Section 3.4 is used to introduce noise to the monolingual data. We randomly sample 1.25 million lines of the union of two output sets (+sample). We filter out sentence pairs in the two output sets with low error rate to make the remaining corpus $E_{\text {rate }}=30 \%$ and randomly sample 1.25 million lines $(+E R 0.3)$. The algorithm 2 is applied on the remaining corpus, making the ratio of coarse-grained error category 1:1:1(+Coarsegained) or the ratio of fine-grained error category 1:1:1:...:1(+Fine-gained).

As it can be seen in Figure 5, the performance of group + sample is much worse than that of group Baseline. This indicates that using the unfiltered data generated by Back-translation is unhelpful for the training of the model. After the sentence pairs with low error rate are filtered out $(+E R 0.3)$, the model score is improved by 0.76 than + sample. Both the result of + Coarse-gained and + Finegained show that the filtered strategy can further improve the score of the model by balancing the proportion of error types in the corpus.

Previous experiments have shown that increasing the number of partial error types leads the model being more inclined to modify specific types of errors, which is the reason for the poor result of group +Sample. The most important contribution of filtering strategy is to keep the same proportion of all error types as possible to improve the performance of the model.

\subsection{Comparison with Other systems}

We use the back-translation method to decode $100 \mathrm{M}$ monolingual data. After filtering out the unqualified data in the synthetic corpus, there was about 10M data left. Then, we synthesize
90M parallel data using rule-based method. All synthetic corpora have about $40 \%$ error rate and the same proportion of error types. In order to generate enough data, we use the monolingual corpus published by Junczys-Dowmunt (JunczysDowmunt and Grundkiewicz, 2016). Neither method of synthesizing data is aimed at spelling errors, so we add a spell checker based on $\mathrm{Cy}$ Hunspell $^{7}$ with a language model training on the monolingual data to correct the spelling errors.

The performance with the well known GEC systems as shown in Table 5. "Data Generation + Transformer" uses about 170 million Wikipedia revision histories with manual modification to pretrain the model. Pre-training the model with synthetic data makes our performance surpass most systems. We only use fewer but more accessible synthetic data to achieve result that is comparable to Lichtarge (Lichtarge et al., 2019).

\section{Discussion}

We performe ablation analysis on our grammatical error correction system. The results of each parts are shown in Table 6.

Only using the Transformer model can make the GEC system perform well. Further use of both large-scale monolingual corpus for data augmentation and model ensemble can greatly improve the performance of the system. Because of the input is token level, Transformer doesn't pay much attention to the information between characters. This allows the spell checker to achieve improvement.

We use the open source tool ERRANT (Bryant et al., 2017) to analyze the $F_{0.5}$ score of the two models for correcting different types of errors. The results of using pre-training data or not are shown in Figure 6, respectively. Comparing the two groups of experiments, we can see that the model pre-training with synthetic data has better performance in most error types than that without using synthetic data. Especially in Verb Inflection (VERB:INFL), Pronoun (PRON), Preposition (PREP), Morphology (MORPH) and Conjunction (CONJ) errors, scores increased by more than 10 percent. However, the model performance declines 5 percent in terms of word order (WO) errors. This may be due to the fact that our data synthesis method does not produce more word order errors. In future experiments, we will consider

\footnotetext{
${ }^{7}$ https://pypi.org/project/CyHunspell/
} 


\begin{tabular}{ccccc}
\hline Model & Learner Corpora & \multicolumn{3}{c}{ CoNLL-2014 } \\
& Precision & Recall & F $_{0.5}$ \\
\hline CNN Seq2Seq(4 ens.+LM+spellcheck) (Chollampatt and Ng, 2018a) & L8,NUCLE & 65.49 & 33.14 & 54.79 \\
Transformer(4 ens.+Many training strategies) (Junczys-Dowmunt et al., 2018) & L8,NUCLE & 63.00 & 38.90 & 56.10 \\
SMT + BiGRU(4 ens.+LM+spellcheck) (Grundkiewicz and Junczys-Dowmunt, 2018) & L8,NUCLE & 66.77 & 34.49 & 56.25 \\
CNN Seq2Seq + Quality Estimation(12 ens.) (Chollampatt and Ng, 2018b) & L8,NUCLE,FCE & - & - & 56.52 \\
Data Generation + Transformer(8 ens.) (Lichtarge et al., 2019) & L8,NUCLE & 66.70 & 43.90 & 60.40 \\
Ours(8 ens.) & L8,NUCLE & $\mathbf{7 0 . 6 3}$ & 37.04 & $\mathbf{5 9 . 7 9}$ \\
\hline
\end{tabular}

Table 5: Comparison of state-of-the-art GEC systems on CoNLL-2014 test set. Our ensemble model is a geometric average of eight best checkpoints for a single model. Here, - represents that the result is not public.

\begin{tabular}{lccc}
\hline Model & $\mathbf{P}$ & $\mathbf{R}$ & $\mathbf{F}_{0.5}$ \\
\hline Transformer & 61.25 & 33.52 & 52.56 \\
+ 100M & 66.64 & 32.65 & 55.16 \\
+ SpellChecker & 67.98 & 34.55 & 56.96 \\
$\quad$ + 8 ens. & 70.63 & 37.04 & 59.79 \\
\hline
\end{tabular}

Table 6: Results on the CoNLL-2014 test sets. $+100 \mathrm{M}$ means using the 100M synthetic data. + SpellChecker refers to add the spelling checker based on the language model mentioned above.

using the method of disrupting the order of sentences.
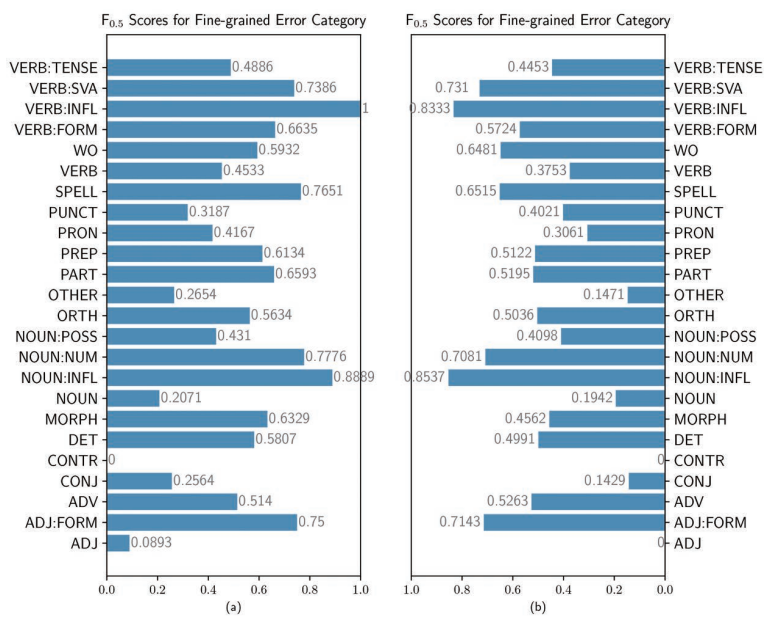

Figure 6: $\mathrm{F}_{0.5}$ scores for Fine-grained Error Category. (a) The model pre-training on the $100 \mathrm{M}$ synthetic data. (b) without pre-training

\section{Related Work}

Felice et al. propose a system that combines a rule-based method and SMT system augmented by a language model (Felice et al., 2014). JunczysDowmunt et al. (Junczys-Dowmunt and Grundkiewicz, 2014) use large-scale Lang- 8 corpus to tune the Moses system (Koehn et al., 2007). These two studies prove that SMT system can combine large corpus and perform well in GEC task. To address the problem of lacking granularity below the word level, Chollampatt uses a character-level SMT to correct the misspelled words (Chollampatt and $\mathrm{Ng}, 2017$ ).

The early work of neural machine translation can not exceed the statistical machine translation at the same period for GEC task (Yuan and Briscoe, 2016; Xie et al., 2016). Chollampatt et al. use a multilayer convolutional encoder-decoder neural network which outperforms the current state-of-the-art statistical machine translation-based approach (Chollampatt and $\mathrm{Ng}, 2018 \mathrm{a}$ ). Grundkiewicz et al. believe that statistical machine translation could not be replaced by deep neural networks. They combine the RNN with a phrase-based SMT system to achieve a higher score (Grundkiewicz and Junczys-Dowmunt, 2018). Junczys-Dowmunt et al. apply Transformer (Vaswani et al., 2017) to the GEC task and achieves good performance (Junczys-Dowmunt et al., 2018).

Motivated by the data scarcity for the GEC task, many researchers focus on generating corpus for data augmentation. Some works use rule-based methods to generate artificial error data that contains specific types of errors (Yuan and Felice, 2013; Xie et al., 2016; Rei et al., 2017). Rozovskaya et al. construct confusion sets to replace specific words in error-free text. There are fewer types of errors covered in synthetic corpus (Rozovskaya and Roth, 2010). Felice et al. use linguistic information to characterize contexts of naturally occurring errors and replicate them in error-free text (Felice and Yuan, 2014). Neither of their methods takes full account of the impact 
of different error rates and error type ratios on the results. Ge et al. propose a dual reinforcement learning strategy that alternately train grammatical error correction and generation model, without using monolingual data (Ge et al., 2018). Unlike them, we use the generation model to decode the monolingual corpus and synthesize a large number of additional grammatical error correction data. Both Rei (Rei et al., 2017) and Xie (Xie et al., 2018) add noise to monolingual data using Backtranslation mechanism of SMT and NMT, respectively. Greedy use of all synthetic corpus makes the models fail to achieve a high score. Lichtarge et al. extract the edits between snapshots from the Wikipedia revisions history to generate parallel corpus (Lichtarge et al., 2019).

\section{Conclusion}

To solve the Grammatical Error Correction problem, we use Transformer which is a powerful deep sequence-to-sequence architecture to translate ungrammatical text into right one. Motivated by the data scarcity for the GEC, we introduce two important concepts, error rate and error type, and propose two data synthesis methods that can control the error rate and ratio of error types on the synthetic data. The first approach is to use different proportions of insert, delete and replace operations to corrupt monolingual corpus. The second approach is to introduce noise into the monolingual corpora by using the Back-translation mechanism and use a controllable method to filter the synthetic parallel data. Both rule-based and Backtranslation methods can be easily applied to any domain or language of monolingual corpora (e.g., biomedical domain, German).

By controlling variables, we validate the effects of different error rates and ratio of error types on the model performance. When the error rate is $40 \%$ and the proportion of different error types is the same, the performance of the model can improve significantly. Our method does not depend on any external knowledge and manual modification, which can achieve comparable score as the state of the art.

Correcting misuse of nouns (NOUN) and verbs (VERB) remains a challenging issue, which requires the understanding of the context and the ability of inference. In the future work, we will try to add some reasoning mechanisms to the sequence-to-sequence model. Grammatical cor- pus will be used to synthesize more word order errors in order to improve the performance on correcting WO errors.

\section{Acknowledgment}

This work is supported by the National Key R\&D Program of China under grant No.2018YFC1900804 and Research Program of State Language Commission under grant No.YB135-89.

\section{References}

Chris Brockett, William B Dolan, and Michael Gamon. 2006. Correcting esl errors using phrasal smt techniques. In Proceedings of the 21st International Conference on Computational Linguistics and the 44th annual meeting of the Association for Computational Linguistics, pages 249-256. Association for Computational Linguistics.

Christopher Bryant, Mariano Felice, and Edward John Briscoe. 2017. Automatic annotation and evaluation of error types for grammatical error correction. Association for Computational Linguistics.

Ciprian Chelba, Tomas Mikolov, Mike Schuster, Qi Ge, Thorsten Brants, Phillipp Koehn, and Tony Robinson. 2013. One billion word benchmark for measuring progress in statistical language modeling. arXiv preprint arXiv:1312.3005.

Shamil Chollampatt and Hwee Tou Ng. 2017. Connecting the dots: Towards human-level grammatical error correction. In Proceedings of the 12th Workshop on Innovative Use of NLP for Building Educational Applications, pages 327-333.

Shamil Chollampatt and Hwee Tou Ng. 2018a. A multilayer convolutional encoder-decoder neural network for grammatical error correction. In ThirtySecond AAAI Conference on Artificial Intelligence.

Shamil Chollampatt and Hwee Tou Ng. 2018b. Neural quality estimation of grammatical error correction. In Proceedings of the 2018 Conference on Empirical Methods in Natural Language Processing, pages 2528-2539.

Daniel Dahlmeier and Hwee Tou Ng. 2012. Better evaluation for grammatical error correction. In Proceedings of the 2012 Conference of the North American Chapter of the Association for Computational Linguistics: Human Language Technologies, pages 568-572. Association for Computational Linguistics.

Daniel Dahlmeier, Hwee Tou Ng, and Siew Mei Wu. 2013. Building a large annotated corpus of learner english: The nus corpus of learner english. In Proceedings of the eighth workshop on innovative use of NLP for building educational applications, pages 22-31. 
Robert Dale, Ilya Anisimoff, and George Narroway. 2012. Hoo 2012: A report on the preposition and determiner error correction shared task. In Proceedings of the Seventh Workshop on Building Educational Applications Using NLP, pages 54-62, Montréal, Canada. Association for Computational Linguistics.

Robert Dale and Adam Kilgarriff. 2011. Helping our own: The hoo 2011 pilot shared task. In Proceedings of the 13th European Workshop on Natural Language Generation, pages 242-249. Association for Computational Linguistics.

Sergey Edunov, Myle Ott, Michael Auli, and David Grangier. 2018. Understanding back-translation at scale. In Proceedings of the 2018 Conference on Empirical Methods in Natural Language Processing, pages 489-500.

Marzieh Fadaee and Christof Monz. 2018. Backtranslation sampling by targeting difficult words in neural machine translation. In Proceedings of the 2018 Conference on Empirical Methods in Natural Language Processing, pages 436-446.

Mariano Felice and Zheng Yuan. 2014. Generating artificial errors for grammatical error correction. In Proceedings of the Student Research Workshop at the 14th Conference of the European Chapter of the Association for Computational Linguistics, pages 116-126.

Mariano Felice, Zheng Yuan, Øistein E. Andersen, Helen Yannakoudakis, and Ekaterina Kochmar. 2014. Grammatical error correction using hybrid systems and type filtering. In Proceedings of the Eighteenth Conference on Computational Natural Language Learning: Shared Task, pages 15-24, Baltimore, Maryland. Association for Computational Linguistics.

Tao Ge, Furu Wei, and Ming Zhou. 2018. Fluency boost learning and inference for neural grammatical error correction. In Proceedings of the 56th Annual Meeting of the Association for Computational Linguistics (Volume 1: Long Papers), pages 10551065 .

Roman Grundkiewicz and Marcin Junczys-Dowmunt. 2018. Near human-level performance in grammatical error correction with hybrid machine translation. In Proceedings of the 2018 Conference of the North American Chapter of the Association for Computational Linguistics: Human Language Technologies, Volume 2 (Short Papers), pages 284-290.

Marcin Junczys-Dowmunt and Roman Grundkiewicz. 2014. The amu system in the conll-2014 shared task: Grammatical error correction by data-intensive and feature-rich statistical machine translation. In Proceedings of the Eighteenth Conference on Computational Natural Language Learning: Shared Task, pages 25-33.
Marcin Junczys-Dowmunt and Roman Grundkiewicz. 2016. Phrase-based machine translation is state-ofthe-art for automatic grammatical error correction. arXiv preprint arXiv:1605.06353.

Marcin Junczys-Dowmunt, Roman Grundkiewicz, Shubha Guha, and Kenneth Heafield. 2018. Approaching neural grammatical error correction as a low-resource machine translation task. In Proceedings of the 2018 Conference of the North American Chapter of the Association for Computational Linguistics: Human Language Technologies, Volume 1 (Long Papers), pages 595-606.

Philipp Koehn, Hieu Hoang, Alexandra Birch, Chris Callison-Burch, Marcello Federico, Nicola Bertoldi, Brooke Cowan, Wade Shen, Christine Moran, Richard Zens, et al. 2007. Moses: Open source toolkit for statistical machine translation. In Proceedings of the 45th annual meeting of the association for computational linguistics companion volume proceedings of the demo and poster sessions, pages $177-180$.

Jared Lichtarge, Chris Alberti, Shankar Kumar, Noam Shazeer, Niki Parmar, and Simon Tong. 2019. Corpora generation for grammatical error correction. arXiv preprint arXiv:1904.05780.

Tomoya Mizumoto, Mamoru Komachi, Masaaki Nagata, and Yuji Matsumoto. 2011. Mining revision log of language learning sns for automated japanese error correction of second language learners. In Proceedings of 5th International Joint Conference on Natural Language Processing, pages 147-155.

Hwee Tou Ng, Siew Mei Wu, Ted Briscoe, Christian Hadiwinoto, Raymond Hendy Susanto, and Christopher Bryant. 2014. The conll-2014 shared task on grammatical error correction. In Proceedings of the Eighteenth Conference on Computational Natural Language Learning: Shared Task, pages 1-14, Baltimore, Maryland. Association for Computational Linguistics.

Hwee Tou Ng, Siew Mei Wu, Yuanbin Wu, Christian Hadiwinoto, and Joel Tetreault. 2013. The conll2013 shared task on grammatical error correction. In Proceedings of the Seventeenth Conference on Computational Natural Language Learning: Shared Task, pages 1-12, Sofia, Bulgaria. Association for Computational Linguistics.

Marek Rei, Mariano Felice, Zheng Yuan, and Ted Briscoe. 2017. Artificial error generation with machine translation and syntactic patterns. In Proceedings of the 12th Workshop on Innovative Use of NLP for Building Educational Applications, pages 287292.

Alla Rozovskaya and Dan Roth. 2010. Generating confusion sets for context-sensitive error correction. In Proceedings of the 2010 Conference on Empirical Methods in Natural Language Processing, pages 961-970. Association for Computational Linguistics. 
Rico Sennrich, Barry Haddow, and Alexandra Birch. 2016a. Improving neural machine translation models with monolingual data. In Proceedings of the 54th Annual Meeting of the Association for Computational Linguistics (Volume 1: Long Papers), volume 1, pages 86-96.

Rico Sennrich, Barry Haddow, and Alexandra Birch. 2016b. Neural machine translation of rare words with subword units. meeting of the association for computational linguistics, 1:1715-1725.

Ashish Vaswani, Noam Shazeer, Niki Parmar, Jakob Uszkoreit, Llion Jones, Aidan N Gomez, Łukasz Kaiser, and Illia Polosukhin. 2017. Attention is all you need. In Advances in neural information processing systems, pages 5998-6008.

Ziang Xie, Anand Avati, Naveen Arivazhagan, Dan Jurafsky, and Andrew Y Ng. 2016. Neural language correction with character-based attention. arXiv preprint arXiv:1603.09727.

Ziang Xie, Guillaume Genthial, Stanley Xie, Andrew $\mathrm{Ng}$, and Dan Jurafsky. 2018. Noising and denoising natural language: Diverse backtranslation for grammar correction. In Proceedings of the 2018 Conference of the North American Chapter of the Association for Computational Linguistics: Human Language Technologies, Volume 1 (Long Papers), pages 619-628.

Zheng Yuan and Ted Briscoe. 2016. Grammatical error correction using neural machine translation. In Proceedings of the 2016 Conference of the North American Chapter of the Association for Computational Linguistics: Human Language Technologies, pages 380-386.

Zheng Yuan and Mariano Felice. 2013. Constrained grammatical error correction using statistical machine translation. In Proceedings of the Seventeenth Conference on Computational Natural Language Learning: Shared Task, pages 52-61.

Wei Zhao, Liang Wang, Kewei Shen, Ruoyu Jia, and Jingming Liu. 2019. Improving grammatical error correction via pre-training a copy-augmente $\mathrm{d}$ architecture with unlabeled data. arXiv preprint arXiv:1903.00138. 